\title{
Conjoint activity of anterior insular and anterior cingulate cortex: awareness and response
}

\author{
Nick Medford • Hugo D. Critchley
}

Received: 19 January 2010/Accepted: 21 April 2010/Published online: 29 May 2010

(C) The Author(s) 2010. This article is published with open access at Springerlink.com

\begin{abstract}
There is now a wealth of evidence that anterior insular and anterior cingulate cortices have a close functional relationship, such that they may be considered together as input and output regions of a functional system. This system is typically engaged across cognitive, affective, and behavioural contexts, suggesting that it is of fundamental importance for mental life. Here, we review the literature and reinforce the case that these brain regions are crucial, firstly, for the production of subjective feelings and, secondly, for co-ordinating appropriate responses to internal and external events. This model seeks to integrate higher-order cortical functions with sensory representation and autonomic control: it is argued that feeling states emerge from the raw data of sensory (including interoceptive) inputs and are integrated through representations in conscious awareness. Correspondingly, autonomic nervous system reactivity is particularly important amongst the responses that accompany conscious experiences. Potential clinical implications are also discussed.
\end{abstract}

Keywords Insula - Anterior cingulate cortex . Functional connectivity - Autonomic nervous system · Subjective awareness

\section{Introduction}

This review considers the joint action of anterior insular cortex (AIC) and anterior cingulate cortex (ACC). There is a large and growing body of evidence that these two

N. Medford $(\bowtie) \cdot$ H. D. Critchley

Department of Psychiatry, Brighton and Sussex Medical School,

Falmer Campus, Brighton BN1 9PX, UK

e-mail: n.medford@bsms.ac.uk regions have a unique functional relationship, which is of relevance as the substrate for a range of experiential and behavioural phenomena, and may hold the key to some of the most elusive questions in modern neuroscience, in particular the question of how subjective experiences (feelings) arise from the raw material of sensory inputs, and how these experiences in turn influence affect, cognition, and behaviour.

The anatomy, physiology, and actions of the AIC are covered at length in other chapters in this special issue and this material will not be duplicated here. But ACC is not specifically covered elsewhere in this issue, so we begin with a brief review of the ACC literature before turning to its joint actions with the insula.

\section{Anatomy of ACC}

The ACC is the anterior part of the cingulate gyrus, a large gyrus situated medially in the cerebral hemisphere that surrounds the corpus callosum ventrally, rostrally, and dorsally. By longstanding convention, cingulate cortex is divided into anterior (ACC), posterior, and retrosplenial regions. The region usually designated as ACC encompasses Brodmann areas 25, 24, and 33, and also includes the caudal part of area 32 (Devinsky et al. 1995). In addition to cortical grey matter, the cingulate gyrus also contains the cingulum, a white matter bundle that runs within the gyrus, crossing into the parahippocampal cortex posteriorly. The cingulum connects different cingulate subregions (see below), and has additional projections into the medial temporal areas (Nieuwenhuys et al. 2008).

A number of schemata are proposed by which the cingulate gyrus may be further divided into anatomical and/or functional subregions. A review of lesion and 
stimulation studies (Vogt et al. 1992) concluded that cingulate cortex as a whole can be divided into an anterior (ACC) region concerned with executive control, particularly of emotion-related processes, and a posterior region specialized for evaluative and monitoring functions. These ideas were extended by an influential review of neuroimaging studies involving ACC activation, which suggested that the region classically designated as ACC can be further subdivided into dorsal cognitive and rostral-ventral affective divisions (Bush et al. 2000).

More recent expert commentary has suggested, on both anatomical and functional grounds, a refinement of the standard anatomical classification, proposing instead a four-region model of cingulate anatomy (Vogt 2005, 2009). In this scheme, the cingulate gyrus is divided into anterior, mid, and posterior cingulate regions, plus a retrosplenial region (located on the ventral surface of the most posterior part of the gyrus). The three main cingulate regions are then further subdivided as follows: anterior cingulate is divided into subgenual (sACC) and pregenual (pACC) areas, mid-cingulate into anterior $(\mathrm{aMCC})$ and posterior (pMCC) areas, and posterior cingulate into dorsal (dPCC) and ventral (vPCC) areas (see Fig. 1). In terms of usefully defining cingulate cortical regions, this model has the advantage of conforming more closely to what is known about cingulate cytoarchitecture and functional anatomy compared with other classifications.

\section{Functions of ACC}

The ACC is implicated as a biological substrate supporting many different mental functions. It is amongst the brain

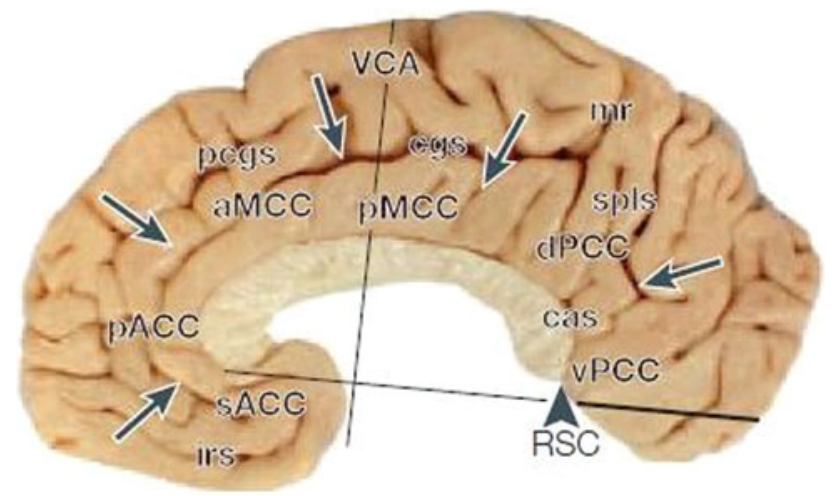

Fig. 1 Subdivisions of cingulate cortex according to the foursubregion model proposed by Vogt (see text). $a M C C$ anterior midcingulate cortex, cas callosal sulcus, cgs cingulate sulcus, $d P C C$ dorsal posterior cingulate cortex, irs inferior rostral sulcus, $m r$ maginal ramus of cgs, $p A C C$ pregenual anterior cingulate cortex, $p c g s$ paracingulate sulcus, $p M C C$ posterior midcingulate cortex, $R S C$ retrosplenial cortex, $s A C C$ subgenual anterior cingulate cortex, spls splenial sulci, $v P C C$ ventral posterior cingulate cortex. (reproduced with permission from Vogt 2005) regions most frequently reported in the functional neuroimaging literature as being significantly activated when engaging in attentionally or behaviourally demanding cognitive tasks (Paus et al. 1998). It is therefore difficult to characterize ACC function in terms of one unifying discrete cognitive operation (although 'attention' was an early contender). Devinsky et al. (1995) observe that "the functions of the anterior cingulate cortex, a component of several vital networks and subserving a diverse range of functions, are difficult to quantify or even describe, and have eluded the traditional 'localized lesion-behavioural correlate' approach". Accounts of ACC function must seek instead to elucidate its role across a wide range of cognitive, affective, and behavioural processes. Although the strength of intrinsic cingulate connections should not be underplayed, different ACC subregions vary in their local and distal anatomical inputs and projections and their cytoarchitectonics, and this is reasonably reflected by the evidence of functional heterogeneity across ACC subregions. For example, in the four-region model of ACC (Vogt 2005) outlined above, the subgenual (sACC) region in particular has close and heavy projections into brain regions typically involved in affective, motivational, and autonomic processing, including amygdala, hypothalamus, and brainstem periaqueductal grey, suggesting an important role in the generation of emotion-related physiological and probably behavioural reactions. Conversely, the posterior midcingulate, which has weaker subcortical connections, is particularly engaged by physically painful stimuli, but not other emotive challenges (Vogt et al. 2003). There are also distinct cingulate motor areas (CMAs), definable by their rich projections into premotor and motor cortices and spinal cord (Morecraft and Tanji 2009). Within the primate forebrain, three CMAs within midcingulate gyrus are identified: a rostral CMA within the anterior MCC, and ventral and dorsal CMAs within the posterior MCC (Dum and Strick 2002). Each CMA receives different thalamic and cortical inputs (Hatanaka et al. 2003) and has different cortical and spinal projections (Dum and Strick 1991), reflected in different functional outputs (Shima et al. 1991).

This degree of functional heterogeneity means that caution is required in making any general statements about the overall function of ACC. Nevertheless, it may be possible to identify common themes across the various aspects of ACC function. The fact that ACC is apparently involved in so many different processes opens the possibility that there is some general, overarching monitoring or control function common to all of them, and/or that ACC is a key area for the integration of ostensibly disparate processes involving affect, cognition, and behaviour. For example, Paus (2001) has suggested that ACC is a region where regulatory and executive processes interact. In this 
model, the outputs of cognitive processing performed elsewhere in the prefrontal cortex are combined in ACC with representations of emotional state to enable appropriate behavioural responses to internal or environmental events.

One influential theory has suggested that ACC is principally involved in error detection (for a discussion of error perception with regard to AIC, see Ullsperger et al. this issue). This idea was based on the discovery of the errorrelated negativity (ERN) potential, an event-related potential consistently observed in experimental trials about $100 \mathrm{~ms}$ following response initiation, when the response is in error (Gehring et al. 1993). This event-related potential was subsequently localized to ACC (Dehaene et al. 1994), leading to the idea that ACC functions could be characterized as the monitoring of, and preparation of response to, errors. However, further studies, reviewed by Paus (2001), suggest that the ERN is not, in fact, error specific, and have also shown that it can be modulated by lesions remote from the ACC. Thus, conceptualization of ACC function as being primarily concerned with error detection is, at best, an incomplete characterization. Even leaving aside the challenge to the idea that comes from empirical studies of the ERN, it is difficult to see how the full range of functions attributed to the ACC could be accommodated within such a concept.

Another line of thought assigns ACC a key role in attentional processes. Mesulam (1981) put forward the idea that ACC is a key part of a cortical network directing the deployment of attentional resources towards external objects, events, and conditions. Within this network, the particular role of ACC was described as being concerned with the assessment of motivational valence, i.e. the motivational significance (in terms of potential reward or punishment) of external stimuli or locations. This implies a role for ACC in the integration of cognitive and affective responses to external events and in the preparation of behavioural responses to those events. These ideas were reformulated and extended by Carter et al. (1999), in a novel theoretical treatment that aimed to reconcile error-detection theory with ideas about ACC and attention. In this model, it is argued that the ACC is concerned with conflict monitoring in a range of different contexts. This includes the online monitoring of responses allowing the identification of errors, as per earlier error-detection theories, and also the detection of conflict between different possible responses to a stimulus, event, or situation. This echoes the idea that ACC is involved in attentional processes, particularly 'attention for action' (Posner et al. 1988). More recently, the conflict-monitoring model has been further revised and extended in an effort to take account of findings related to an apparent role for ACC in decision making (Botvinick 2007) (see also Nelson et al. this issue).
A stance taken from observing the close correspondence between autonomic output and cingulate function across a range of tasks provided a view consistent with the notion of cingulate as 'limbic motor cortex' (Craig 2002). In this 'unified' model (Critchley et al. 2003; Critchley 2004), the emphasis was placed on the (presumed facilitatory) control of autonomic state as the major cortical output of ACC, accommodating subregional differences in both the types of information processed (motivational in genual/subgenual, cognitive in dorsal anterior midcingulate subregions) and in the autonomic response axes (sympathetic in dorsal ACC, parasympathetic/antisympathetic in subgenual ACC). Ultimately, this model attempted to reconcile the mass of data from imaging and related studies, highlighting ACC involvement with the relative lack of marked cognitive (including attentional or executive) or physical disability, other than with self-initiated behaviour or sustained performance, consequent on acquired ACC damage (see below). This model has been empirically qualified (Critchley et al. 2005b), but also specifically criticized (Bush 2009). Nevertheless, the notion that internal state is fundamental to cognition and motivation requires a neural mediator, and perhaps one that is intrinsic to the mammalian brain. The detailed relationship between cingulate activity and behaviourally evoked changes in bodily state continues to be substantiated (e.g. Wager et al. 2009a, b; see also Lane 2008). The link with other automatic processes such as attentional direction and salience processing (Seeley et al. 2007) is suspected to be intrinsic to the conjoint functioning of ACC and insular cortex (Craig 2009) and their phylogenetic evolution in primates.

The developmental trajectory of such ideas is of interest: it can be seen that to take account of the multiplicity of findings related to functions of the ACC, it has been necessary for theorists to repeatedly 'zoom out' (i.e. to conceive the role of ACC in ever broader terms). The implication is that ACC function may in fact be fundamental to many different aspects of mental life.

Similar comments may be made about the functions of the AIC. Craig (2009) lists a dizzying range of contexts in which AIC activation has been reported in functional neuroimaging studies, before suggesting that the way to reconcile these disparate findings is to posit the AIC as the seat of consciousness itself: the neural correlate of firstperson subjective awareness. Craig also notes that the great majority of studies reporting AIC activation also report activation of ACC. This is particularly true of studies involving any aspect of emotional processing (Craig 2009) and leads logically to the idea that it may be productive to consider the two regions together when attempting to generate more powerfully explanatory models of their function. This approach is explored in detail below, but first we will briefly consider the effects of lesions of the 
ACC. This data can further inform our understanding of ACC function and is particularly pertinent to the ensuing discussion of the inter-relationship of ACC and AIC.

\section{Effects of ACC lesions}

As might be predicted from above, lesions involving the ACC have been linked with a wide range of cognitive, affective, and behavioural sequelae in both animals and humans. Unilateral cingulate lesions in monkeys produce contralateral motor neglect (Watson et al. 1973), and bilateral lesions in humans have been reported to cause akinetic mutism (Nielsen and Jacobs 1951; Nemeth et al. 1988). Kennard (1955) briefly reviews the early animal and human ACC lesion literature (noting reports of apathy, akinetic mutism, and changes in motor behaviour and vocalization) before presenting findings from cingulate ablation studies in cats and monkeys. Post-surgery, the animals were passive and hypomotile. In addition, cats showed a range of autonomic and behavioural changes, becoming apparently more emotionally aroused as reflected by behavioural changes, such as increased purring and growling (though notably the latter was not associated with any increase in physical aggression, the animals were described as growling more readily in response to minor stimuli, yet never engaging in actual combat with other cats).

With regard to mutism (see above), an authoritative recent review of vocalization in primates (Jurgens 2009) presents evidence that ACC is crucial in the voluntary initiation of vocalization, through connections with brainstem periaqueductal grey (Vogt and Vogt 2009). This in turn connects with brainstem nuclei that innervate the muscles of articulation and phonation. ACC does not, however, appear to be required for simple vocalization responses to basic stimuli. The idea that ACC is implicated in higher-level voluntary control of responses is consistent with data from human studies suggesting that ACC has a key role in response initiation, across a wide range of contexts (see below for further discussion of this). In view of our theme of ACC-AIC interaction, it should also be noted that a number of studies of speech production in humans have highlighted the left AIC as being involved in the preparation and co-ordination of articulation and phonation (Blank et al. 2002; Ackermann and Riecker 2004; Riecker et al. 2005).

During the period that the early ACC lesion studies were conducted, there was also a surge of interest in the potential use of cingulate surgery as a therapeutic intervention in intractable psychiatric disorder. Reported outcomes of these procedures tended to be largely favourable (Kennard 1955). Tow and Whitty (1953) describe mental and behavioural changes following bilateral suction-ablation of BA 24 (anterior midcingulate in the four-region model) in patients suffering from mixed anxiety and depression with obsessive-compulsive features. In most cases, cingulate surgery was associated with subsequent clinical improvement, with post-operative patients generally described as less troubled by obsessional symptoms, more relaxed and socially confident, and more emotionally expressive. In some cases, there were negative aspects to these changes. Increased irritability, evidence of impaired judgement, and diminished drive were described in a number of cases, including some where surgery was overall judged to have been a success in terms of symptom reduction. It is noteworthy that both the positive and negative changes seen in these patients have common features, apparently reflecting a reduction in drive, a relaxation of emotional control, and a reduced concern about the social judgements or emotional responses of others. Bearing in mind our overall theme of conjoint AIC and ACC activity, it is of interest that a study of the neuropsychiatric sequelae of insular stroke found a high incidence of abulia and anergia, with reduced motivation and drive (Manes et al. 1999). The authors speculate that this may reflect the disconnection of insula from ACC.

Advances in neurosurgery, particularly the development of stereotactic techniques, have refined cingulate surgery, allowing more precise localization of intervention. Targeted stereotactic cingulotomy remains a viable treatment in severe intractable conditions of the type outlined above, and has also been used in chronic pain syndromes and in the treatment of substance addiction (Brotis et al. 2009). A more recent survey of patients who underwent cingulotomy for chronic pain noted subtle impairments in the spontaneous initiation of behaviour and the formation of intentions (Cohen et al. 1999), comparable to the older findings outlined above. However, a review of cingulotomy outcome in chronic refractory depression concluded that the most favourable clinical outcomes were obtained with more anterior lesions within the midcingulate region. Posterior midcingulate lesions were less clinically effective and may fall outside the area of cingulate gyrus most commonly reported as showing abnormal activity in functional neuroimaging studies of major depression (Steele et al. 2008).

The specificity of lesion effects in different parts of the ACC is emphasized by a report of a patient with a wellcircumscribed ACC lesion. The patient showed evidence of impairment on divided-attention tasks when required to give manual responses, but not when giving vocal responses (Turken and Swick 1999). This is further evidence that the ACC is involved in response selection and initiation, and that the functional organization of these roles is structured enough for the occurrence of modality- 
specific deficits. This report is unusual in describing a lesion confined exclusively to ACC. The majority of the ACC lesion literature reports findings in patients with damage to ACC and adjacent structures in the medial frontal lobe (Devinsky et al. 1995). In particular, the supplementary motor area (SMA) is frequently affected, making it difficult to determine the precise contribution of cingulate cortex disruption to the observed deficits. However, the idea that ACC is involved in response generation is supported by studies in which ACC damage was associated with abnormalities of motor activity. In particular, in the 'alien hand syndrome' (AHS) there are involuntary exploratory, grasping, and manipulation movements of one hand. Feinberg et al. (1992) argue that there are two distinct subtypes of AHS, which they designate as frontal and callosal on the basis of the site of damage. Frontal AHS is associated with ACC damage, although this was only one of the medial prefrontal areas implicated. An increase in manual manipulation behaviour has also been reported in macaques following bilateral cingulate lesioning (Hadland et al. 2003). There is also a report of a patient who developed contralateral paroxysmal AHS following ischaemic damage to the rostral CMA (Brazdil et al. 2006). In addition, a study of pathological grasping phenomena showed that ACC damage was more strongly associated with such phenomena than damage to any other prefrontal area (De Renzi and Barbieri 1992). However, another study suggested that ACC damage was specifically associated with the emergence of pathological groping behaviours rather than grasping (Hashimoto and Tanaka 1998). This discrepancy notwithstanding, it appears that ACC damage is associated with abnormal and involuntary motor responses, leading to the suggestion (Paus 2001) that ACC damage predisposes to the emergence of uncontrolled motor programmes or 'subroutines' that are normally suppressed.

ACC dysfunction may have implications for social functioning: Hadland et al. (2003) noted decreased sociability in macaques following bilateral cingulate lesions; a human lesion study of note is that by Baird et al. (2006). These authors report findings in three patients, with damage to the medial frontal areas principally involving genual ACC (BA 24, 32), who were tested on tasks designed to probe social cognition, including tests of emotional facial expression recognition and theory of mind. Perhaps surprisingly, only subtle deficits were identified, with evidence of theory-of-mind impairment in only one patient, who differed from the other two in having bilateral lesions. Two of these patients had previously been identified as having impaired autonomic function (Critchley et al. 2003, see below), which might be expected to lead to deficits in social cognition. The authors suggest that the neural circuitry underlying theory of mind and other aspects of social cognition is sufficiently widely distributed such that social deficits may only become apparent following more extensive structural damage.

We noted above that theories, which attempt to give an account of ACC action in terms of specific cognitive functions (such as error detection or conflict monitoring), struggle to take account of other findings. This point is underlined by a study of four patients with damage to the dorsal ACC who were tested on cognitive tasks involving a high level of response conflict (e.g. a go/no-go task, in which the frequency of no-go stimuli was varied to allow comparison between high and low conflict conditions) (Fellows and Farah 2005). Patients did not differ from controls on measures of cognitive control, as indexed by relative change in reaction time and error rate between low and high conflict tasks. The authors suggest that this implies that ACC is not, in fact, essential for cognitive control. However, it was also the case that ACC patients were significantly slower than controls in responding, across all tasks and conditions. This suggests that ACC damage does, in fact, impair the initiation of responses, even if the pattern of this impairment is not what might be predicted from cognitive theories of ACC function. Again, this raises the issue that ACC appears to be involved in response selection and preparation, but that it does not seem to be possible to form an adequate characterization of this involvement in terms of specific cognitive actions. Another important insight into ACC function comes from an fMRI study exploring autonomic changes during the performance of tasks involving cognitive effort in healthy controls and three patients with ACC damage (Critchley et al. 2003). All three patients showed diminished autonomic cardiovascular responses to cognitive effort when compared with 147 normal controls. In addition, the sympathetic component of heart rate variability was found to correlate with changes in neural activity in both ACC and insula, lending weight to the idea that these two brain regions are concerned with, and responsive to, the regulation of bodily states and the generation of physiological arousal states appropriate to behavioural and environmental demands. With this in mind, it is of note that studies in animals have repeatedly found evidence of impaired autonomic responses following ACC ablation (Devinsky et al. 1995). The role of ACC and insula with respect to autonomic function is explored further below.

Finally, whilst not strictly related to lesion studies, it should also be noted that ACC is implicated in the pathophysiology of obsessive-compulsive disorder (OCD). In addition to numerous functional neuroimaging studies (reviewed in Saxena et al. 2009) reporting abnormal patterns of ACC activity in OCD, there is converging evidence of abnormal ACC morphology (Radua and Mataix-Cols 2009; Shim et al. 2009) and neurochemistry (Ebert et al. 
1997; Rosenberg et al. 2004; Arnold et al. 2009) in this condition. Precise elucidation of ACC involvement in OCD is still awaited, but will probably require an approach which recognizes that different ACC subregions are likely to have more relevance to different clinical subtypes (e.g. washing, checking, hoarding) (Saxena et al. 2009; Middleton 2009).

\section{Joint action of ACC and AIC}

For the remainder of this review, ACC function will be considered in tandem with the functions of the AIC. It will be argued that this conjoint approach can provide an illuminating perspective on the functions of these regions, as they appear to constitute input (AIC) and output (ACC) components of a system based on awareness of self: that is, an integrated awareness of cognitive, affective, and physical state, generated by the integrative functions of the AIC and then re-represented in ACC as a basis for the selection of, and preparation for, responses to inner or outer events. These responses are not necessarily motor: examples of non-motor responses might include a shift of cognitive set during task performance (Dosenbach et al. 2006), or a topdown modulation of sensory processing. Before proceeding with this analysis, however, we will briefly review the anatomical connections and evidence of functional connectivity between these two regions.

In primates, the cingulate cortex, particularly the area corresponding to BA 24, has reciprocal connections with both granular and dysgranular layers of the insular cortex (Mesulam and Mufson 1982; Vogt and Pandya 1987; Nieuwenhuys et al. 2008; Yukie and Shibata 2009). This reciprocity is significant: whilst our general argument is that insula integrates sensory information into awareness and that this information is then transferred to $\mathrm{ACC}$, it should also be noted that back-projections from the ACC may in theory allow the insular representation of the feeling state to be modulated by cingulate activity. Both AIC and ACC contain populations of von Economo neurons, and Craig (2009) has suggested that it is these neurons which form the connections between these areas. (crossrefs). A study of visceral pain (Moisset et al. 2010) successfully used diffusion tensor imaging to demonstrate tracts connecting the two regions. Other evidence of a direct functional relationship comes from an fMRI study of resting state connectivity, in which fluctuations in BOLD signal are correlated between regions. This study suggested that, in humans, there are two insula-cingulate systems, with anterior insula showing functional connectivity with anterior and midcingulate regions, and $\mathrm{mid} /$ posterior insula connected with only the posterior midcingulate region (Taylor et al. 2009). This work complements a previous study of intrinsic connectivity, which suggested that both ACC and insula are core regions of a 'salience network', responsive to a wide range of stimuli relevant to emotional and/or motivational states. It is functionally linked to subcortical structures also involved in such responses, such as the extended amygdala, dorsomedial thalamus, hypothalamus, and periaqueductal grey (Seeley et al. 2007). Dosenbach et al. $(2006,2007)$ report that ACC and AIC are crucially implicated in the establishment and maintenance of task set, whilst Harrison et al. (2008) found that the connectivity of these areas increased during the induction of sadness, supporting the idea that their joint activity is a key element of the neural basis of emotional experience. Whilst these studies do not directly demonstrate information exchange between AIC and ACC, they strongly suggest a high degree of functional connectivity between the regions. More specific evidence of functional interaction between ACC and AIC comes from a study by Sridharan et al. (2008), which identified a fronto-insular cingulate system involved in switching between default mode and central executive networks. The application of Granger causality analysis suggested direct causal interactions between activity in the right fronto-insular cortex (rFIC) and other cortical areas across a range of tasks. The activity in ACC shows the strongest such interaction, suggesting a powerful causal effect for rFIC in determining activity in ACC. There was also evidence of a weaker causal interaction in the opposite direction. An intriguing recent finding is that resting state functional connectivity between AIC and rostral ACC is negatively correlated with subclinical autistic traits in healthy adults (Di Martino et al. 2009). This hints at the role of this system in self-related processes, a theme explored in detail below.

With regard to self-related processes and AIC, influential ideas, highly relevant to our argument here, have been developed by Damasio, whose 'neural self' concept (e.g. Damasio 2003) postulates the insula as crucial to the generation of subjective feeling states. In this model, neural information from afferent somatosensory pathways is integrated into somatotopic representations in the posterior insula before being 're-represented' in mid- and anterior insula. This 're-representation' entails integration of the sensory information with other aspects of conscious experience to produce a continuous, but fluctuating, stream of awareness and feeling. The key idea is that information derived from the body forms the basis of feeling states and emotional experience, although this does not necessarily require the insula to be the source of awareness itself.

A term such as 'neural self' begs questions of definition, but the concept of 'self' eludes precise definition. Any attempt at such definition inevitably opens up whole territories of scientific and philosophical uncertainty: the nature of first-person subjective awareness or the validity 
and meaning of lived experience. Scientific, clinical, and philosophical investigations of the self utilize widely differing terminologies and theoretical frameworks, such that the different perspectives may confuse as much as inform each other (Northoff and Heinzel 2003). Furthermore, the self may be as much a socio-cultural construct as a measurable psychobiological entity (Berrios and Markova 2003), although it is reasonable to suppose that selfconcepts across different cultures will tend to involve at least some common elements.

Damasio offers no formal definition of 'self' but argues instead for a 'core self': a mental representation corresponding to our innate, moment-to-moment feeling of subjective awareness. This awareness has both continuity and individuality: "to a first approximation, the self is a stable representation of individual continuity which serves as a mental reference for the organism within the conscious mind" (Damasio 2003).

How does this relate to more abstract concepts such as personhood or identity? Damasio reserves the term 'autobiographical self' for these more complex notions, but nevertheless argues that the 'core' neural self must be the foundation of more elaborated conceptions of self and personal identity.

If the 'core' self is rooted in information carried by afferent somatosensory systems, it is important to examine the mechanisms by which we sense ourselves. There are so many that Damasio suggests a new classification of the senses is required to take account of them. In his proposed scheme, the conventional five senses are classed as 'exteroceptive', whilst vestibular, pain and temperature, and proprioceptive systems are 'interoceptive', combining to produce interoception: a 'sense of the internal milieu' (Craig 2002). Direct evidence for the idea that the insula is a key area supporting interoception comes from a functional neuroimaging study in which the ability to make accurate judgements about the timing of one's own heartbeat was used as an index of interoceptive awareness (Critchley et al. 2004). Activity in the right anterior insula was found to predict accuracy of performance on the heartbeat task, whilst activity in the dorsal ACC increased significantly during attention to the internal physical state.

The conceptual link between interoception and emotional experience is supported by a significant body of converging empirical data. Studies by Feldman Barrett et al. (2004) and Pollatos et al. (2007) have shown a correlation between interoceptive awareness and self-reported emotional experience. In the latter study, high-density EEG was used to localize electrophysiological responses to emotionally salient pictures, with both AIC and ACC emerging as key areas engaged in such responses. The authors interpreted this as demonstrating involvement of both 'first-order' (AIC) and 'second-order' (ACC) response areas, arguing that AIC activity reflects the representation of bodily state as a feeling, and that ACC "may serve as an interface structure for translating emotional reactions into differential somatic responses" (Pollatos et al. 2007). This interpretation is consistent with the ideas presented here. Further support comes from an fMRI study showing that AIC and dorsal ACC activity were associated with heart rate changes in response to emotional facial expressions (Critchley et al. 2005a). Also a study showed that bilateral AIC and rostral ACC were amongst brain areas showing a significant response to errors on a numerical Stroop-type task, and were also activated by sympathetic arousal, indexed by pupillometry measures taken concurrently with task performance. In addition, activity in a cingulate area spanning pregenual and anterior midcingulate regions was predictive of variability in autonomic arousal within individual participants (Critchley et al. 2005b). This constitutes powerful evidence that dorsal ACC is crucial in the generation of autonomic responses to internal and external events (see Fig. 2).

A number of functional neuroimaging studies have examined regional brain activity associated with interoceptive awareness of oesophageal or gastric distention. Positron emission tomography (PET) studies by Stephan et al. (2003) and Vandenbergh et al. (2005) found AIC and ACC activation as part of a network of brain regions responsive to gastric distention, although in the former study the ACC activation was localized to subgenual ACC, whereas the latter study reported activation of anterior midcingulate (BA 24). This latter is consistent with two studies that have manipulated task conditions to probe the neural correlates of gastro-intestinal sensation in more detail. Phillips et al. (2003) studied responses to oesophageal distention coupled with the viewing of neutral or fearful facial expressions, designed to provide an emotional context that would modulate sensory processing. Bilateral insula and ACC were amongst brain regions activated in response to oesophageal stimulation, and midcingulate gyrus (BA 24) and bilateral AIC were significantly more active when oesophageal stimulation occurred in a negative emotional context (and were the only brain regions to show this response pattern, making this a particularly striking finding). Coen et al. (2007) manipulated the intensity of oesophageal stimulation to enable comparison between non-painful and painful conditions. Bilateral AIC and right ACC were amongst the brain areas activated in the nonpainful condition, and bilateral AIC and bilateral ACC in the painful condition. Of particular note was the finding that activity in midcingulate gyrus (BA 24) correlated with subjective ratings of pain. Pain may be considered as an interoceptive feeling (Craig 2009), but one that has its own extensive literature, which is briefly reviewed here with regard to AIC and ACC. 

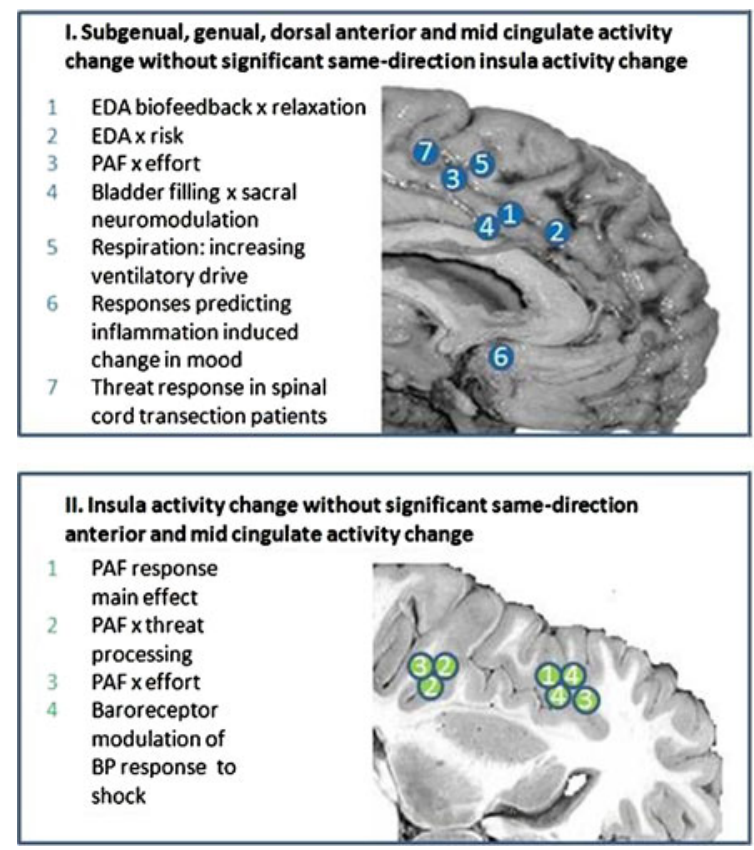

Fig. 2 Anterior cingulate-insula relationships in behaviourally integrated autonomic control. This figure plots locations of dissociated and conjoint activations of anterior/mid/subgenual cingulate and insular cortical activity across experimental studies involving the Critchley laboratory exploring central correlates of peripheral autonomic activity during different task conditions, representation/ perturbation of afferent visceral feedback or both (biofeedback, baroreceptor or autonomic patient vs. control studies). The figure does not explore lateralization of insular response with the observation that across studies activity was generally bilateral or right lateralized. The figure highlights a paucity of cingulate or insula-only engagement and notes the contexts in which these are observed. While not a

\section{Pain}

Since the nineteenth century, it has been known from animal studies that cingulate lesions produce a decrease in pain sensitivity and avoidance (Devinsky et al. 1995; Lawson et al. 2009). In humans, stereotactic cingulotomy is used as a treatment for refractory chronic pain (Brotis et al. 2009). The localization and functional specialization of nociceptive areas within cingulate gyrus is reviewed in detail by Vogt (2005) and forms part of the rationale for his four-region model of cingulate anatomy, outlined above. However, it is important to note that no cingulate subregion is uniquely concerned with pain response: rather it appears that cingulate regions subserve a range of processes, which are particularly relevant to pain (Vogt and Sikes 2009). Cingulate pain responses are concentrated in the pregenual and midcingulate regions (Vogt 2005) and have generally been thought to subserve affective-motivational aspects of pain (Price 2000), an interpretation that sits well with the more general role that ACC plays in emotion. However, a recent expert review (Vogt and Sikes 2009) highlights that not all findings relating

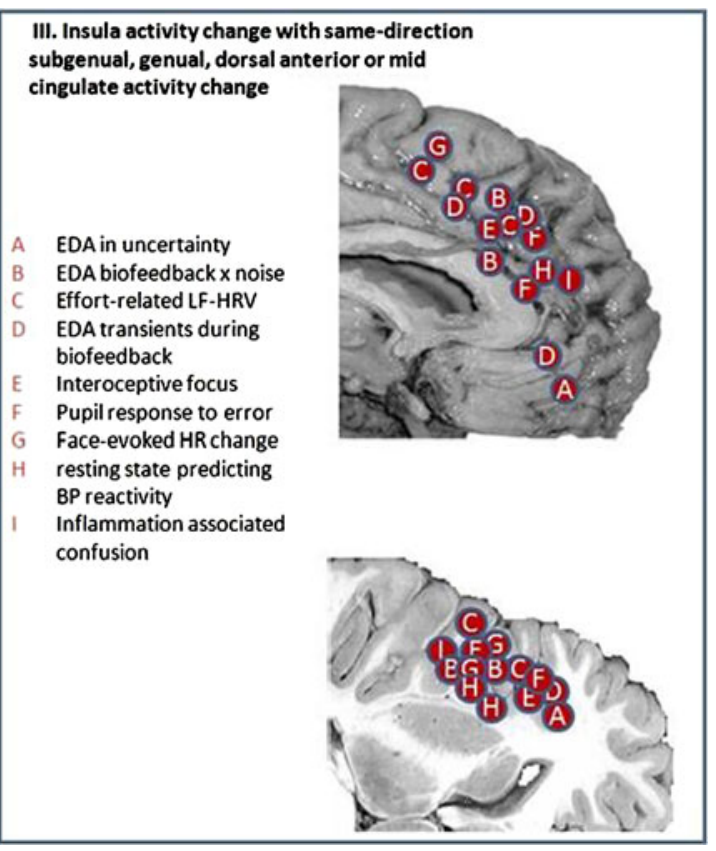

comprehensive view of coupling between these regions, the figure focuses directly on the control of internal bodily state. EDA electrodermal activity, $P A F$ pure autonomic failure, $B P$ blood pressure, $L F-H R V$ low frequency (sympathetic) power in heart rate variability, $H R$ heart rate. Panel I 1 Critchley et al. 2001a, 2 Critchley et al. 2001b, 3 Critchley et al. 2001c, 4 Kavia et al. 2009, 5 McKay et al. 2010, 6 Harrison et al. 2009a, 7 Nicotra et al. 2006. Panel II 1 Critchley et al. 2001c, 2 Critchley et al. 2002a, 3 Critchley et al. 2001c, 4 Gray et al. 2009. Panel III A Critchley et al. 2001b, B Critchley et al. 2002b, C Critchley et al. 2003, D Nagai et al. 2004, E Critchley et al. 2004, F Critchley et al. 2005b, G Critchley et al. 2005a, H Gianaros et al. 2009, I Harrison et al. 2009b

to ACC and pain can be readily integrated into an affectivemotivational model, and proposes a "cingulate premotor pain model" in which tiers of processing involving cingulate cortex facilitate autonomic and behavioural responses to noxious stimuli. Motor responses here include not only avoidance behaviours, but also facial expression (grimacing, wincing, etc.) and vocalization (see above).

ACC and insula are key regions of the medial pain system, which receives inputs from midline and intralaminar thalamic nuclei [the lateral nuclei project into lateral pain system areas, principally primary and secondary somatosensory cortices (Petrovic et al. 1999; Vogt 2005)]. Given this, it is unsurprising that functional neuroimaging studies implicate both ACC and anterior insula in the cortical response to pain (Craig 2009). A PET study of responses to painful stimulation of the dorsum of the foot found that ipsilateral AIC and contralateral ACC were significantly more active in the pain condition than at rest (Andersson et al. 1997). However, a PET study of mechanical allodynia in five patients with peripheral mononeuropathy found that bilateral AIC and bilateral 
ACC activity showed co-variance with pain intensity across the experimental group (Petrovic et al. 1999). Another PET study used intradermal capsaicin to induce pain and allodynia. The authors identified a widely distributed response network, which they interpreted as reflecting four aspects of pain-related processing: sensory perception, attention, top-down modulation, and sensorimotor integration (Iadorola et al. 1998). Within this framework, they argue that the insula is involved in both perceptual and sensorimotor aspects, and that ACC is primarily involved in attention to pain. This may be consistent with ideas regarding the role of ACC in attention (see above). In the light of other evidence discussed above, we would suggest that ACC involvement in the pain response is not simply attentional, but also reflects the preparation and initiation of autonomic and motor responses to pain.

It is a long-established finding that painful stimuli evoke two distinct sensory experiences, known as first and second pain. Using magnetoencephalography (MEG), a method which has the advantage of high temporal resolution, Ploner et al. (2002) demonstrated that the timing of pregenual ACC activity in response to painful stimuli suggests a specific involvement in second pain, which is thought to reflect affective and motivational aspects of pain: awareness of unpleasant sensation and preparation of avoidance responses. This finding is complemented by evidence from animal studies that the ACC contains a final representation of affective-motivational elements of pain (Sewards and Sewards 2002, but see also comments above). PET studies in humans show that components of the medial pain system, including pregenual and anterior midcingulate, are selectively activated by attention to unpleasantness of pain, rather than by attention to the location of the painful stimulus (Kulkarni et al. 2005). Also, ACC responses to pain can be modulated by hypnotic suggestions designed to alter the unpleasantness, but not the intensity, of pain (Rainville et al. 1997). A PET study of neural responses to graded intensities of pain found that insula and ACC were co-activated by increasing stimulus intensity (Derbyshire et al. 1997), whilst ACC ablation studies in rodents indicate that lesioning of rostral ACC in rats produces a decrease in pain avoidance behaviours (Johansen et al. 2001). Taken together, these findings suggest that AIC is involved in awareness of the unpleasant feeling associated with pain, and that ACC is involved in the marshalling of response to this unpleasantness. This is consistent with our proposed overall scheme of AIC and ACC function.

\section{Emotional awareness and awareness of self and moment}

Co-activation of the AIC and ACC is reported by numerous functional neuroimaging studies of emotional processing
(Craig 2009). If the insular cortex is implicated in the generation of feeling states, and the ACC in both directing attention (see above) and the facilitation of autonomic responses, it is reasonable to predict that both areas will be engaged by tasks involving the direction of attention towards induced emotion (Lane 2008). An early PET study in which participants were asked to attend to subjective emotional responses supports this: attention to emotion was associated with activation of both ACC and AIC (Lane et al. 1997). A more recent fMRI study in which participants viewed alternating blocks of aversive and neutral images (McRae et al. 2008) reinforces this finding. Activity in the dorsal ACC and left insular cortex whilst viewing highly arousing images was significantly related to individual subject emotional awareness as measured by the Levels of Emotional Awareness Scale (Lane et al. 1990). The authors also measured skin conductance responses as an index of autonomic arousal during scanning blocks. They found a significant relationship between these responses and activity in the dorsal ACC, supporting the idea that this region of ACC was crucially involved in the generation of autonomic responses to emotionally salient stimuli.

In the light of the above findings regarding emotional awareness, deficits of such awareness are also of interest here (Lane 2008). A number of studies have examined the neural response to emotionally salient stimuli in individuals with alexithymia, defined as a reduced capacity for emotionalizing, fantasizing, and identifying, verbalizing, and thinking about emotions (Bermond et al. 2006). Functional neuroimaging studies of alexithymia have consistently shown reduced activation of ACC in response to emotionally relevant stimuli (e.g. Kano et al. 2003; Moriguchi et al. 2007; Frewen et al. 2008; Karlsson et al. 2008; see also Bermond et al. 2006), but findings with regard to the insula have been more variable, with some studies reporting increased insula activation in alexithymics in response to emotional material, and some reporting decreases (Craig 2009). However, of note, one study in particular that found increased insula activation (Karlsson et al. 2008) did not distinguish between anterior and posterior insula in reporting and discussing this finding, but the reported cluster of increased insula activation in fact fell within the right posterior insula rather than AIC. A similar finding, although on the left side, was reported by Frewen et al. (2008) in a study of alexithymia in the context of post-traumatic stress disorder (PTSD). The same study found reduced activation in AIC (and ACC) bilaterally in the same participants during the same experimental condition. Thus, in alexithymia there may be opposite response patterns in the posterior and anterior insular cortices. This is a speculative suggestion; however, it is difficult to know how far findings in PTSD can be generalized to 
alexithymia as a whole. However, a study by Moriguchi et al. (2007) found increased activity in both anterior and posterior insula (both right-sided) in alexithymics, making empathic judgements of pain in others a finding that appears inconsistent with the suggestion above.

If is true that the insula is involved in the generation of all subjective feeling states, and that the combined action of AIC and ACC provides the neural basis of self-awareness, then it may be possible to identify conjoint AIC and ACC activity related to 'awareness of the moment' and 'awareness of self' (Craig 2009). There is partial support for the former idea from an fMRI study using an attentional blink paradigm to assess the neural correlates of stimulus awareness (Kranczioch et al. 2005). In each trial of this paradigm, two targets (T1 and T2) are presented, separated by a brief temporal interval. By manipulating the duration of this interstimulus interval, it is possible to create conditions where T2 is not consciously perceived (i.e. an attentional blink occurs), and compare with conditions where it is perceived. Brain regions that were significantly more active when T2 was perceived included the left ACC and an area that the authors localized to the inferior frontal gyrus, but which may also include the left AIC. This line of research is linked to work on the ACC and error detection (see above) by a recent fMRI study of error awareness (Klein et al. 2007). In this study, participants performed a visual saccade task in which it was possible to be either aware or unaware of errors. Anterior midcingulate gyrus, SMA, and bilateral AIC were significantly activated in association with errors, but only AIC was activated when participants signalled awareness of such errors.

With regard to awareness of self, a recent fMRI study of self-recognition provides strong support for the involvement of the right AIC and ACC. The study showed significant activation during self-related processing, whether subjects were viewing pictures of their own faces or other body parts (Devue et al. 2007). The authors conclude that specific insular and cingulate subregions are key areas for integrative self-related processing. This interpretation is in line with other studies that have probed self-awareness and the subjective experience of self-agency (see Northoff and Bermpohl 2004, for a review). Further evidence comes from a more recent fMRI study, which found activation in ACC and left AIC during self-reflection, the latter region being uniquely activated by self-reflection amongst a range of tasks performed (Modinos et al. 2009). A relatively little-studied psychiatric condition, depersonalization disorder, provides an intriguing clinical perspective on these findings, explored below.

It is reasonable to suppose that mechanisms supporting self-awareness may also be important for understanding the mental states of others, and a number of studies have reported joint activation of AIC and ACC by tasks designed to elicit empathic responses (Lieberman 2007). A study by Singer et al. (2004) is of particular note: AIC and ACC were amongst a network of regions activated by a task eliciting empathy for the pain of others. Significantly, these were the only regions in the network in which activity correlated with a measure of self-reported empathy. If these regions are crucial for empathy, then they play a key role in a wide range of social interactions, with relevance for personality typology and the emerging field of social neuroscience (Lieberman 2007).

\section{Clinical contexts}

Given the mass of evidence that AIC and ACC are both crucially implicated in emotional processing and first-person subjective experience, it is reasonable to suppose that functional abnormalities of these regions may be involved in the pathophysiology of psychiatric disorders. Influential models of mood disorder suggest that the neurobiological basis of major depression is dysfunction of the normal interactions between limbic and cortical areas (Mayberg 1997; Drevets et al. 2008), although these models implicate widely distributed networks and are not specific to the regions under consideration here. However a recent metaanalysis of anatomical findings in schizophrenia and bipolar disorder found that grey matter abnormalities in bilateral insula and ACC are the most commonly reported such abnormalities in bipolar disorder (Ellison-Wright and Bullmore 2010).

The interoceptive functions of AIC and ACC may be particularly relevant to anxiety states, which are characterized not only by cognitive features such as worry, but also by physical changes such as increased heart rate and motor restlessness. These changes are experienced as an unpleasant core element of the anxious state. Paulus and Stein (2006) have put forward a theoretical account of anxiety emphasizing the role of the insula in the generation of feeling states and awareness of bodily sensations. A recent review of neuroimaging studies in anxiety and phobic disorders concluded that AIC and ACC, along with the amygdala, are the brain regions most commonly implicated in such studies (Damsa et al. 2009).

Because ACC is known to be involved in motor response selection and initiation, it is possible to extend these ideas to disorders involving abnormal movements. In particular, the tics seen in Gilles de la Tourette syndrome and related disorders lend themselves to this analysis, since they are frequently preceded by uncomfortable inner sensations (premonitory urges), and tic occurrence is associated with a subjective sense of relief from such sensations. A reasonable hypothesis will be that functional abnormalities in the AIC-ACC system are involved in the 
production of a sense of inner tension and premonitory urge (AIC) and in the generation of abnormal movements and/or vocalizations (ACC; a similar idea in a non-clinical context was suggested by a study, which found bilateral insula and ACC activation associated with blink suppression in healthy adults (Lerner et al. 2009). Here, one can hypothesize that AIC represents the 'urge to blink' feeling, and that ACC is involved in suppressing or overriding this urge). A PET study of tics in Tourette syndrome (Stern et al. 2000) found that activity in both AIC and ACC was significantly correlated with tic occurrence, although many other areas were similarly correlated, making specific interpretations difficult. Intriguingly, however, a recent structural MRI study of brain morphology in familial cases of Tourette syndrome reported reduced cortical thickness of the frontal motor, cingulate, and insular cortices, with the degree of cortical thinning correlated with tic severity. The authors suggest that in Tourette syndrome, there may be delayed or abnormal maturation of areas involved in self-regulation. This predisposes to motor and vocal tics and to other failures of self-regulation, such as impulsive aggression (Fahim et al. 2009).

Depersonalization disorder (DPD) is a relatively littlestudied condition that may be particularly relevant here. DPD is characterized by a pervasive and disturbing sense of unreality in both experience of self (depersonalization) and surroundings (derealization) (Medford et al. 2005; Sierra 2009). The phenomenology of DPD is complex, encompassing abnormalities of bodily sensation (typically, reduced sensation or even a sense of complete disembodiment) and emotional experience (diminished subjective and autonomic responsivity to emotional stimuli or events, perhaps implying some overlap with the concept of alexithymia, discussed above) (Sierra et al. 2002, 2005; Simeon et al. 2008). Thus, in addition to measurable autonomic abnormalities suggesting a role for ACC, the symptoms of DPD can be construed as pathological variants of 'awareness of self' and 'awareness of the moment', concepts already linked to conjoint AIC and ACC action. Findings from neuroimaging studies lend support to this idea. Phillips et al. (2001) found that DPD patients showed significantly less activation in the left insula and bilateral ACC compared to normal controls when viewing aversive images. However, a study of verbal emotional memory in DPD showed that DPD patients show little difference in the neural response to emotional and neutral blocks of a verbal recognition memory task. This was in contrast to controls, in whom there were extensive emotion-related activations including AIC and ACC (Medford et al. 2006). Future studies combining functional neuroimaging and autonomic measurements may be of particular value in DPD.

ACC and AIC are also likely to be involved in the experience of physical illness. Studies of pain and discomfort induced by gastro-intestinal stimulation (see above) have some relevance here, but the experience of feeling unwell has been assessed more directly by a recent study examining neural activity associated with an inflammatory response induced by typhoid vaccine (Harrison et al. 2009b). Activity associated with inflammation was seen in a network of cortical and subcortical structures, including ACC and insula. It was interpreted as reflecting "the integration of affective and motivational processing with afferent interoceptive information" (Harrison et al. $2009 \mathrm{~b}$ ) and also the initiation of autonomic responses to the physiological disturbance. Again, this speaks of the idea that the insula and ACC represent the afferent and efferent arms of a system for regulating physical states and for generating subjective experiences (feelings) on the basis of those states.

\section{Conclusion}

The multiplicity of contexts in which AIC/ACC conjoint action has been observed underlines the idea that these regions constitute a functional system engaged in a great many aspects of mental life. It is possible to extrapolate these empirical findings into bold theoretical positions with relevance to both science and philosophy. These ideas can furnish novel perspectives on the aetiology and management of neuropsychiatric disorders and provide an exciting framework for understanding the relationship between neurobiology and conscious experience. Future work may focus on more precise delineation of the functional relationship between AIC, ACC, and associated brain systems. In particular, it is anticipated that the study of the integration of autonomic activity with higher-order cortical representations will yield important insights into fundamental questions of basic and clinical neuroscience.

Open Access This article is distributed under the terms of the Creative Commons Attribution Noncommercial License which permits any noncommercial use, distribution, and reproduction in any medium, provided the original author(s) and source are credited.

\section{References}

Ackermann H, Riecker A (2004) The contribution of the insula to motor aspects of speech production: a review and a hypothesis. Brain Lang 89:320-328

Andersson JL, Lilja A, Hartvig P, Langstrom B, Gordh T, Handwerker H, Torebjork E (1997) Somatotopic organization along the central sulcus for pain localization in humans as revealed by positron emission tomography. Exp Brain Res 117:192-199

Arnold PD, Macmaster FP, Richter MA, Hanna GL, Sicard T, Burroughs E, Mirza Y, Easter PC, Rose M, Kennedy JL, Rosenberg DR (2009) Glutamate receptor gene (GRIN2B) associated with reduced anterior cingulate glutamatergic 
concentration in pediatric obsessive-compulsive disorder. Psychiatry Res 172:136-139

Baird A, Dewar BK, Critchley H, Dolan R, Shallice T, Cipolotti L (2006) Social and emotional functions in three patients with medial frontal lobe damage including the anterior cingulate cortex. Cogn Neuropsychiatry 11:369-388

Bermond B, Vorst HC, Moormann PP (2006) Cognitive neuropsychology of alexithymia: implications for personality typology. Cogn Neuropsychiatry 11:332-360

Berrios G, Markova IS (2003) The self and psychiatry: a conceptual history. In: Kircher T, David A (eds) The self in neuroscience and psychiatry. Cambridge University Press, Cambridge

Blank SC, Scott SK, Murphy K, Warburton E, Wise RJ (2002) Speech production: Wernicke, Broca and beyond. Brain 125:1829-1838

Botvinick MM (2007) Conflict monitoring and decision making: reconciling two perspectives on anterior cingulate function. Cogn Affect Behav Neurosci 7:356-366

Brazdil M, Kuba R, Rektor I (2006) Rostral cingulate motor area and paroxysmal alien hand syndrome. J Neurol Neurosurg Psychiatry 77:992-993

Brotis AG, Kapsalaki EZ, Paterakis K, Smith JR, Fountas KN (2009) Historic evolution of open cingulotomy and stereotactic cingulotomy in the management of medically intractable psychiatric disorders, pain and drug addiction. Stereotact Funct Neurosurg 87:271-291

Bush G (2009) Dorsal anterior midcingulate cortex: roles in normal cognition and disruption in attention-deficit/hyperactivity disorder. In: Vogt BA (ed) Cingulate neurobiology and disease. Oxford University Press, UK, pp 245-274

Bush G, Luu P, Posner MI (2000) Cognitive and emotional influences in anterior cingulate cortex. Trends Cogn Sci 4:215-222

Carter CS, Botvinick MM, Cohen JD (1999) The contribution of anterior cingulate cortex to executive processes in cognition. Rev Neurosci 10:49-57

Coen SJ, Gregory LJ, Yaguez L, Amaro E, Brammer M, Williams SCR, Aziz Q (2007) Reproducibility of human brain activity evoked by esophageal stimulation using functional magnetic resonance imaging. Am J Physiol Gastrointest Liver Physiol 293:188-197

Cohen RA, Kaplan RF, Moser DJ, Jenkins MA, Wilkinson H (1999) Impairments of attention after cingulotomy. Neurology $53: 819-824$

Craig AD (2002) How do you feel? Interoception: the sense of the physiological condition of the body. Nat Rev Neurosci 3:655666

Craig AD (2009) How do you feel-now? The anterior insula and human awareness. Nat Rev Neurosci 10:59-70

Critchley HD (2004) The human cortex responds to an interoceptive challenge. Proc Natl Acad Sci USA 101:6333-6334

Critchley HD, Melmed RN, Featherstone E, Mathias CJ, Dolan RJ (2001a) Brain activity during biofeedback relaxation: a functional neuroimaging investigation. Brain 124:1003-1012

Critchley HD, Mathias CJ, Dolan RJ (2001b) Neural activity in the human brain relating to uncertainty and arousal during reward anticipation. Neuron 29:537-545

Critchley HD, Mathias CJ, Dolan RJ (2001c) Neural correlates of first and second-order representation of bodily states. Nature Neurosci 4:207-212

Critchley HD, Mathias CJ, Dolan RJ (2002a) Fear-conditioning in humans: the influence of awareness and arousal on functional neuroanatomy. Neuron 33:653-663

Critchley HD, Melmed RN, Featherstone E, Mathias CJ, Dolan RJ (2002b) Volitional control of autonomic arousal: a functional magnetic resonance study. Neuroimage 16:909-919

Critchley HD, Matthias CJ, Joesphs O, O'Doherty J, Zanini S, Dewar B-K, Cipolotti L, Shallice T, Dolan RJ (2003) Human cingulate cortex and autonomic control: converging neuroimaging and clinical evidence. Brain 126:2139-2152

Critchley HD, Wiens S, Rotshtein P, Ohman A, Dolan RJ (2004) Neural systems supporting interoceptive awareness. Nat Neurosci 7:189-195

Critchley HD, Rotshtein P, Nagai Y, O'Doherty J, Mathias CJ, Dolan RJ (2005a) Activity in the human brain predicting differential heart rate responses to emotional facial expressions. Neuroimage 24:751-762

Critchley HD, Tang J, Glaser D, Butterworth B, Dolan RJ (2005b) Anterior cingulate activity during error and autonomic response. Neuroimage 27:885-895

Damasio A (2003) Feelings of emotion and the self. Ann NY Acad Sci 1001:253-261

Damsa C, Kosel M, Moussally J (2009) Current status of brain imaging in anxiety disorders. Curr Opin Psychiatry 22:96-110

De Renzi E, Barbieri CA (1992) The incidence of the grasp reflex following hemispheric lesion and its relation to frontal damage. Brain 115:293-313

Dehaene S, Posner MI, Tucker DM (1994) Localization of a neural system for error detection and compensation. Psychol Sci 5:303305

Derbyshire SWG, Jones AKP, Gyulai F, Clark S, Townsend D, Firestone LL (1997) Pain processing during three levels of noxious stimulation produces differential patterns of central activity. Pain 73:431-445

Devinsky O, Morell MJ, Vogt BA (1995) Contributions of anterior cingulate cortex to behaviour. Brain 118:279-306

Devue C, Collette F, Balteau E, Degueldre C, Luxen A, Maquet P, Breart S (2007) Here I am: the cortical correlates of visual selfrecognition. Brain Res 1143:169-182

Di Martino A, Shehzad Z, Kelly C, Roy AK, Gee DG, Uddin LQ, Gotimer K, Klein DF, Castellanos FX, Milham MP (2009) Relationship between cingulo-insular functional connectivity and autistic traits in neurotypical adults. Am J Psychiatry 166: 891-899

Dosenbach NUF, Visscher KM, Palmer ED, Miezin FM, Wenger KK, Hyunseon CK, Burgund ED, Grimes AL, Schlaggar BL, Petersen SE (2006) A core system for the implementation of task sets. Neuron 50:799-812

Dosenbach NUF, Fair DA, Miezin FM, Cohen AL, Wenger KK, Dosenbach RAT, Fox MD, Snyder AZ, Vincent JL, Raichle ME, Schlaggar BL, Petersen SE (2007) Distinct brain networks for adaptive and stable task control in humans. Proc Natl Acad Sci USA 104:11073-11078

Drevets WC, Price JL, Furey ML (2008) Brain structural and functional abnormalities in mood disorders: implications for neurocircuitry models of depression. Brain Struct Funct 213:93-118

Dum RP, Strick PL (1991) The origin of corticospinal projections from the premotor areas in the frontal lobe. J Neurosci 11:667-689

Dum RP, Strick PL (2002) Motor areas in the frontal lobe of the primate. Physiol Behav 77:677-682

Ebert D, Speck O, Konig A, Gerger M, Hennig J, Hohagen F (1997) $1 \mathrm{H}$-magnetic resonance spectroscopy in obsessive-compulsive disorder: evidence for neuronal loss in the cingulate gyrus and the right striatum. Psychiatry Res 74:173-176

Ellison-Wright I, Bullmore E (2010) Anatomy of bipolar disorder and schizophrenia: a meta-analysis. Schizophr Res 117:1-12

Fahim C, Yoon U, Sandor P, Frey K, Evans AC (2009) Thinning of the motor-cingulate-insular cortices in siblings concordant for Tourette syndrome. Brain Topogr 22:176-184

Feinberg TE, Schindler RJ, Flanagan NG, Haber LD (1992) Two alien hand syndromes. Neurology 42:19-24

Feldman Barrett L, Quigley KS, Bliss-Moreau AronsonKR (2004) Interoceptive sensitivity and self-reports of emotional experience. J Pers Soc Psychol 87:684-697 
Fellows LK, Farah MJ (2005) Is anterior cingulate cortex necessary for cognitive control? Brain 128:788-796

Frewen PA, Lanius RA, Dozois DJ, Neufeld RW, Pain C, Hopper JW, Densmore M, Stevens TK (2008) Clinical and neural correlates of alexithymia in posttraumatic stress disorder. J Abnorm Psychol 117:171-181

Gehring WJ, Goss B, Coles MGH, Meyer DE, Donchin E (1993) A neural system for error detection and compensation. Psychol Sci 4:385-390

Gianaros PJ, Sheu LK, Remo AM, Christie IC, Critchley HD, Wang JJ (2009) Resting perfusion of corticolimbic brain areas predicts individual differences in stressor-evoked blood pressure reactivity. Hypertension 53:819-825

Gray MA, Rylander K, Harrison NA, Wallin BG, Critchley HD (2009) Following one's heart: cardiac rhythms gate central initiation of sympathetic reflexes. J Neurosci 29:1817-1825

Hadland KA, Rushworth MFS, Gaffan D, Passingham RE (2003) The effect of cingulate lesions on social behaviour and emotion. Neuropsychologia 41:919-931

Harrison BJ, Pujol J, Ortiz H, Fornito A, Pantelis C, Yucel M (2008) Modulation of brain resting-state networks by sad mood induction. PLoS One 3:1-12

Harrison NA, Brydon L, Walker C, Gray MS, Steptoe A, Critchley HD (2009a) Inflammation causes mood change through alterations in subgenual cingulate activity and mesolimbic connectivity. Biol Psych 66:407-414

Harrison NA, Brydon L, Walker C, Gray M, Dolan RJ, Steptoe A, Critchley HD (2009b) Neural origins of human sickness in interoceptive responses to inflammation. Biol Psych 66:415-422

Hashimoto R, Tanaka Y (1998) Contribution of the supplementary motor area and anterior cingulate gyrus to pathological grasping phenomena. Eur Neurol 40:151-158

Hatanaka N, Tokuno H, Hamada I, Inase M, Ito Y, Imanishi M, Hasegawa N, Akazawa T, Nambu A, Takada M (2003) Thalamocortical and intracortical connections of monkey cingulate motor areas. J Comp Neurol 462:121-138

Iadorola MJ, Berman KF, Zeffiro TA, Byas-Smith MG, Gracely RH, Max MB, Bennett GJ (1998) Neural activation during acutecapsaicin-evoked pain and allodynia assessed with PET. Brain 121:931-947

Johansen JP, Fields HL, Manning BH (2001) The affective component of pain in rodents: direct evidence for a contribution of the anterior cingulate cortex. Proc Natl Acad Sci USA 98:80778082

Jurgens U (2009) The neural control of vocalization in mammals: a review. J Voice 23:1-10

Kano M, Fukudo S, Gyoba J, Kamachi M, Tagawa M, Mochizuki H, Itoh M, Hongo M, Yanai K (2003) Specific brain processing of facial expressions in people with alexithymia: an H2 15O-PET study. Brain 126:1474-1484

Karlsson H, Naatanen P, Stenman H (2008) Cortical activation in alexithymia as a response to emotional stimuli. Br J Psychiatry 192:32-38

Kavia R, DasGupta R, Critchley H, Fowler CJ, Griffiths D (2009) An fMRI study of the effect of neuromodulation on brain responses in women with Fowler's Syndrome. BJU Int 105:336-372

Kennard MA (1955) The cingulate gyrus in relation to consciousness. J Nerv Ment Dis 121:34-39

Klein TA, Endrass T, Kathmann N, Neumann J, von Cramon DY, Ullsperger M (2007) Neural correlates of error awareness. Neuroimage 34:1774-1781

Kranczioch C, Debener S, Schwarzbach J, Goebel R, Engel AK (2005) Neural correlates of conscious perception in the attentional blink. Neuroimage 24:704-714

Kulkarni B, Bentley DE, Elliott R, Youell P, Watson A, Derbyshire SWG, Frackowiak RSJ, Friston KJ, Jones AKP (2005) Attention to pain localization and unpleasantness discriminates the functions of the medial and lateral pain systems. Eur $\mathbf{J}$ Neurosci 21:3133-3142

Lane RD (2008) Neural substrates of implicit and explicit emotional processes: a unifying framework for psychosomatic medicine. Psychosom Med 70:214-231

Lane RD, Quinlan DM, Schwartz GE, Walker PA, Zeitlin SB (1990) The levels of emotional awareness scale: a cognitive-developmental theory and its application to psychopathology. J Pers Assess 55:124-134

Lane RD, Fink GR, Chua P, Dolan RJ (1997) Neural activation during selective attention to subjective emotional responses. Neuroreport 8:3969-3972

Lawson HC, Ohara S, Greenspan JD, Coghill RC, Lenz FA (2009) Neurophysiology of cingulate pain responses and neurosurgical pain interventions. In: Vogt BA (ed) Cingulate neurobiology and disease. Oxford University Press, UK, pp 401-417

Lerner A, Bagic A, Hanakawa T, Boudreau EA, Pagan F, Mari Z, Bara-Jimenez W, Aksu M, Sato S, Murphy DL, Hallett M (2009) Involvement of insula and anterior cingulate cortices in control and suppression of natural urges. Cereb Cortex 19:218-223

Lieberman MD (2007) Social cognitive neuroscience: a review of core processes. Annu Rev Psychol 58:259-289

Manes F, Paradiso S, Robinson RG (1999) Neuropsychiatric effects of insular stroke. J Nerv Ment Dis 187:707-712

Mayberg HS (1997) Limbic-cortical dysregulation: a proposed model of depression. J Neuropsychiatry Clin Neurosci 9:471-481

McKay LC, Critchley HD, Murphy K, Frackowiak RSJ, Corfield DR (2010) Sub-cortical and brainstem sites associated with chemostimulated increases in ventilation in humans. Neuroimage 49:2526-2535

McRae K, Reiman EM, Fort CL, Chen K, Lane RD (2008) Association between trait emotional awareness and dorsal anterior cingulate activity during emotion is arousal-dependent. Neuroimage 41:648-655

Medford N, Sierra M, Baker D, David AS (2005) Understanding and treating depersonalisation disorder. Adv Psychiatr Treat 11:92100

Medford N, Brierley B, Brammer M, Bullmore ET, David AS, Phillips ML (2006) Emotional memory in depersonalization disorder: a functional MRI study. Psychiatry Res 148:93-102

Mesulam MM (1981) A cortical network for directed attention and unilateral neglect. Ann Neurol 10:309-325

Mesulam MM, Mufson EJ (1982) Insula of the old world monkey: III. Efferent cortical output and comments on function. J Comp Neurol 212:38-52

Middleton FA (2009) The contribution of anterior cingulate-basal ganglia circuitry to complex behaviour and psychiatric disorders. In: Vogt BA (ed) Cingulate neurobiology and disease. Oxford University Press, UK, pp 619-632

Modinos G, Ormel J, Aleman A (2009) Activation of anterior insula during self-reflection. PLoS One 4:e4618

Moisset X, Bouhassira D, Ducreux D, Glutron D, Coffin B, Sabate JM (2010) Anatomical connections between brain areas activated during rectal distension in healthy volunteers: a visceral pain network. Eur J Pain 14:142-148

Morecraft RJ, Tanji J (2009) Cingulofrontal interactions and the cingulate motor areas. In: Vogt BA (ed) Cingulate neurobiology and disease. Oxford University Press, UK, pp 113-144

Moriguchi Y, Decety J, Ohnishi T, Maeda M, Mori T, Nemoto K, Matsuda H, Komaki G (2007) Empathy and judging others' pain: an fMRI study of alexithymia. Cereb Cortex 17:2223-2234

Nagai Y, Critchley HD, Featherstone E, Trimble MR, Dolan RJ (2004) Activity in ventromedial prefrontal cortex covaries with sympathetic skin conductance level: a physiological account of a "default mode" of brain function. Neuroimage 22:243-251 
Nemeth G, Hegedus K, Molnar L (1988) Akinetic mutism associated with bicingular lesions: clinicopathological and functional anatomical correlates. Eur Arch Psychiatry Neurol Sci 237:218-222

Nicotra A, Critchley HD, Mathias CJ, Dolan RJ (2006) Emotional and autonomic consequences of spinal cord injury explored using functional brain imaging. Brain 129:718-728

Nielsen JM, Jacobs LJ (1951) Bilateral lesions of the anterior cingulate gyri. Bull Los Angeles Neuro Soc 16:231-234

Nieuwenhuys R, Voogd J, van Huijzen C (2008) The human central nervous system, 4th edn. Springer, Heidelberg

Northoff G, Bermpohl F (2004) Cortical midline structures and the self. Trends Cogn Sci 8:102-107

Northoff G, Heinzel A (2003) The self in philosophy, neuroscience and psychiatry: an epistemic approach. In: Kircher T, David A (eds) The self in neuroscience and psychiatry. Cambridge University Press, Cambridge

Paulus MP, Stein MB (2006) An insular view of anxiety. Biol Psychiatry 60:383-387

Paus T (2001) Primate anterior cingulate cortex: where motor control, drive and cognition interface. Nat Rev Neurosci 2:417-424

Paus T, Koski L, Caramanos Z, Westbury C (1998) Regional differences in the effects of task difficulty and motor output on blood flow response in the human anterior cingulate cortex: a review of 107 PET activation studies. NeuroReport 9:R37-R47

Petrovic P, Ingvar M, Stone-Elander S, Petersson KM, Hansson P (1999) A PET activation study of dynamic mechanical allodynia in patients with mononeuropathy. Pain 83:459-470

Phillips ML, Medford N, Senior C, Bullmore ET, Suckling J, Brammer MJ, Andrew C, Sierra M, Williams SCR, David AS (2001) Depersonalization disorder: thinking without feeling. Psychiatry Res 108:145-160

Phillips ML, Gregory LJ, Cullen S, Coen S, Ng V, Andrew C, Giampietro V, Bullmore E, Zelaya F, Amaro E, Thompson DG, Hobson AR, Williams SCR, Brammer M, Aziz Q (2003) The effect of negative emotional context on neural and behavioural responses to oesophageal stimulation. Brain 126:669-684

Ploner M, Gross J, Timmermann L, Schnitzler A (2002) Cortical representation of first and second pain sensation in humans. Proc Natl Acad USA 99:12444-12448

Pollatos O, Gramann K, Schandry R (2007) Neural systems connecting interoceptive awareness and feelings. Hum Brain Mapp 28:9-18

Posner MI, Petersen SE, Fox PT, Raichle ME (1988) Localization of cognitive operations in the human brain. Science 240:1627-1631

Price DD (2000) Psychological and neural mechanisms of the affective dimension of pain. Science 288:1769-1772

Radua J, Mataix-Cols D (2009) Voxel-wise meta-analysis of grey matter changes in obsessive-compulsive disorder. Br J Psychiatry 195:393-402

Rainville P, Duncan GH, Price DD, Carrier B, Bushnell MC (1997) Pain affect encoded in human anterior cingulate but not somatosensory cortex. Science 277:968-971

Riecker A, Mathiak K, Wildgruber D, Erb M, Hertrich I, Grodd W, Ackermann H (2005) fMRI reveals two distinct cerebral networks subserving speech motor control. Neurology 64:700-706

Rosenberg DR, Mirza Y, Russell A, Tang J, Smith JM, Banerjee SP, Bhandari R, Rose M, Ivey J, Boyd C, Moore GJ (2004) Reduced anterior cingulate glutamatergic concentrations in childhood OCD and major depression versus healthy controls. J Am Acad Child Adolesc Psychiatry 43:1146-1153

Saxena S, O'Neill J, Rauch SL (2009) The role of cingulate cortex dysfunction in obsessive-compulsive disorder. In: Vogt BA (ed) Cingulate neurobiology and disease. Oxford University Press, UK, pp 587-617

Seeley WW, Menon V, Schatzberg AF, Keller J, Glover GH, Kenna $\mathrm{H}$, Reiss AL, Greicius MD (2007) Dissociable intrinsic connectivity networks for salience processing and executive control. J Neurosci 27:2349-2356

Sewards TV, Sewards MA (2002) The medial pain system: neural representations of the motivational aspect of pain. Brain Res Bull 59:163-180

Shim G, Jung WH, Choi JS, Jung MH, Jang JH, Park JY, Choi CH, Kang DH, Kwon JS (2009) Reduced cortical folding of the anterior cingulate cortex in obsessive-compulsive disorder. J Psychiatry Neurosci 34:443-449

Shima K, Aya K, Mushiake H, Inase M, Aizawa H, Tanji J (1991) Two movement-related foci in the primate cingulate cortex observed in signal-triggered and self-paced forelimb movements. J Neurophysiol 65:188-202

Sierra M (2009) Depersonalization: a new look at a neglected syndrome. Cambridge University Press, Cambridge

Sierra M, Senior C, Dalton J, McDonough M, Bond A, Phillips ML, O'Dwyer AM, David AS (2002) Autonomic response in depersonalization disorder. Arch Gen Psychiatry 59:833-838

Sierra M, Baker D, Medford N, David AS (2005) Unpacking the depersonalization syndrome: an exploratory factor analysis on the Cambridge Depersonalization Scale. Psychol Med 35:15231532

Simeon D, Kozin DS, Segal K, Lerch B, Dujour R, Giesbrecht T (2008) Deconstructing depersonalization: further evidence for symptom clusters. Psychiatry Res 157:303-306

Singer T, Seymour B, O’Doherty J, Kaube H, Dolan RJ, Frith CD (2004) Empathy for pain involves the affective but not sensory components of pain. Science 303:1157-1162

Sridharan D, Levitin DJ, Menon V (2008) A critical role for the right fronto-insular cortex in switching between central-executive and default-mode networks. Proc Natl Acad Sci USA 105:12569_ 12574

Steele JD, Christmas D, Eljamel MS, Matthews K (2008) Anterior cingulotomy for major depression: clinical outcome and relationship to lesion characteristics. Biol Psychiatry 63:670-677

Stephan E, Pardo JV, Faris PL, Hartman BK, Kim SW, Ivanov EH, Daughters RS, Costello PA, Goodale RL (2003) Functional neuroimaging of gastric distention. J Gastrointest Surg 7:740-749

Stern E, Silbersweig DA, Chee KY, Holmes A, Robertson MM, Trimble M, Frith CD, Frackowiak RS, Dolan RJ (2000) A functional neuroanatomy of tics in Tourette syndrome. Arch Gen Psychiatry 57:741-748

Taylor KS, Seminowicz DA, Davis KD (2009) Two systems of resting state connectivity between the insula and cingulate cortex. Hum Brain Mapp 30:2731-2745

Tow PM, Whitty CWM (1953) Personality changes after operations on the cingulate gyrus in man. J Neurol Neurosurg Psychiatry $16: 186-193$

Turken AU, Swick D (1999) Response selection in the human anterior cingulate cortex. Nat Neurosci 2:920-924

Vandenbergh J, Dupont P, Fischler B, Bormans G, Janssens J, Track J (2005) Regional brain activation during proximal stomach distention in humans. Gastroenterology 128:564-573

Vogt BA (2005) Pain and emotion interactions in subregions of the cingulate gyrus. Nat Rev Neurosci 6:533-544

Vogt BA (2009) Regions and subregions of the cingulate cortex. In: Vogt BA (ed) Cingulate neurobiology and disease. Oxford University Press, UK, pp 3-30

Vogt BA, Pandya DN (1987) Cingulate cortex of the rhesus monkey: II. Cortical afferents. J Comp Neurol 262:271-289

Vogt BA, Sikes RW (2009) Cingulate nociceptive circuitry and roles in pain processing: the cingulate premotor pain model. In: Vogt BA (ed) Cingulate neurobiology and disease. Oxford University Press, UK, pp 311-338

Vogt BA, Vogt LJ (2009) Mu-opioid receptors, placebo map, descending systems, and cingulate-mediated control of 
vocalization and pain. In: Vogt BA (ed) Cingulate neurobiology and disease. Oxford University Press, UK, pp 311-338

Vogt BA, Finch DM, Olson CR (1992) Functional heterogeneity in cingulate cortex. Cereb Cortex 2:435-443

Vogt BA, Berger GR, Derbyshire SWG (2003) Structural and functional dichotomy of human midcingulate cortex. Eur J Neurosci 18:3134-3144

Wager TD, Waugh CE, Lindquist M, Noll DC, Fredrickson BL, Taylor SF (2009a) Brain mediators of cardiovascular responses to social threat: part I. Reciprocal dorsal and ventral sub-regions of the medial prefrontal cortex and heart-rate reactivity. Neuroimage 47:821-835
Wager TD, van Anst VA, Hughes BL, Davidson ML, Lindquist MA, Ochsner KA (2009b) Brain mediators of cardiovascular responses to social threat: part II. Prefrontal-subcortical pathways and relationship with anxiety. Neuroimage 47:836851

Watson RT, Heilman KM, Cauthen JC, King FA (1973) Neglect after cingulectomy. Neurology 23:1003-1007

Yukie M, Shibata H (2009) Temporocingulate interactions in the monkey. In: Vogt BA (ed) Cingulate neurobiology and disease. Oxford University Press, UK, pp 145-162 